\author{
Enzo Neppi* \\ Université Grenoble Alpes
}

\title{
CORINNE OU L'ITALIE DI MADAME DE STAËL: I VICOLI CIECHI DELL'INCONTRO CULTURALE E AMOROSO FRA LE 'NAZIONI' NELL'EUROPA DEL PRIMO OTTOCENTO
}

\begin{abstract}
La posizione di Madame de Staël sul problema del carattere nazionale va inserita in un dibattito che vede la partecipazione di numerosi intellettuali europei fin dalla metà del Settecento - un dibattito in cui lingua, letteratura e politica appaiono strettamente connesse. Nelle sue principali opere, da De la littérature a De l'Allemagne, Madame de Staël presenta posizioni che sono nel complesso coerenti e costanti, ma arricchite da sottili distinguo. In Corinne, in particolare, il contrasto tra i caratteri nazionali diviene un ostacolo che neppure l'amore fra gli individui riesce a superare, ma il dialogo fra le nazioni e i caratteri è ribadito come un valore, preferibile, almeno idealmente, al ripiegamento sulla propria identità nazionale.
\end{abstract}

Parole chiave: Madame de Staël, carattere nazionale, patriottismo, incontro tra culture, rappresentazione dell'amore, Corinne ou l'Italie.

\section{IL CARATTERE NAZIONALE, DA MONTESQUIEU A FOSCOLO}

In un breve saggio del 1748, Of National Characters, David Hume (1987) negava l'influenza del clima e di altre cause fisiche sui caratteri delle nazioni, e si opponeva in particolare alla tesi secondo cui le nazioni avrebbero caratteri permanenti, che si manifestano con costanza nel corso dei secoli. Il caso suo era tuttavia un'eccezione. Proprio negli anni in cui il filosofo inglese avanzava questa prudente opinione, si diffondeva in Europa - e prima di tutto in Francia, culla dei Lumi, in autori come Du Bos, Buffon, Montesquieu e Voltaire ${ }^{1}$ - l'idea che ogni popolo abbia un suo

*enzo.neppi@univ-grenoble-alpes.fr

${ }^{1}$ Vedi, per questi autori, e per le successive osservazioni sulla filosofia della lingua in Germania, le acute analisi di Crépon (1996: 39-63, 89, 121-155, 195-326). 
specifico carattere nazionale, determinato da un lato da cause fisiche, come il clima e la razza, e dall'altro da «cause morali»: la lingua, i costumi, la religione e le leggi. Gli illuministi francesi potevano essere in disaccordo sull'importanza e sul peso relativo di ciascuna di queste cause. Ma che le nazioni abbiano un genio, un'indole, un carattere innato sembrava a tutti, o a quasi tutti, evidente.

In contrasto con la tesi diffusa in Francia sulla superiore universalità della lingua francese, la cui sintassi sarebbe più fedele alla forma logica del pensiero, e sarebbe determinata non dalle passioni ma dalla ragione (vedi in particolare Rivarol 1995: 162), in Germania, nei medesimi anni, Klopstock e Lessing riprendono una difesa della lingua tedesca in cui si era impegnato già Leibniz a partire dagli anni ' 80 del XVII secolo. Per tale via essi ovviamente reagiscono ai modelli letterari più in voga in Francia, e che si erano imposti per un certo tempo anche nel loro paese, e esprimono il loro rifiuto degli orientamenti più razionalisti e materialisti dell'illuminismo francese. Da parte sua, Herder ha una vasta visione delle lingue e delle culture e pensa che ogni nazione contribuisca individualmente, attraverso la propria storia, al rivelarsi della provvidenza divina nel mondo. Ma nel medesimo tempo è anche convinto che un legame profondo unisca ogni popolo alla sua lingua, e di conseguenza difende il «pregiudizio», il «nazionalismo» [Nationalism], che riconducendo i popoli al loro centro [Mittelpunkt], stringendoli al loro ceppo, esigendo la fedeltà alla propria lingua e cultura, li rende più ardenti e felici (Herder 1994: 39-40). In questa prospettiva la lingua è un tesoro, un debito della nazione verso se stessa, e il principale dovere di ogni scrittore consiste nel rimanere fedele al genio della propria lingua e al carattere della propria nazione.

Pur presentandosi a prima vista come l'affermazione di un fatto empirico, la nozione di carattere nazionale acquista dunque molto rapidamente un carattere normativo, e diventa, in questo doppio registro, una sorta di a priori epocale, una premessa sistemica che dominerà a lungo il discorso europeo sulle nazioni e sulle culture ${ }^{2}$. Gli obblighi del cittadino verso la propria patria si configurano allora come un dovere supremo, che esige una dedizione totale ed esclusiva, e tende a sostituirsi alle credenze religiose tradizionali.

In Italia la svolta epocale che rende possibili simili affermazioni sul carattere nazionale della letteratura è attestata dal contrasto (contrasto ideologico, ma che tende a diventare anche uno spartiacque generazionale)

${ }^{2}$ Mi riferisco qui agli studi di Alberto Mario Banti, e in particolare a Banti (2005) e, per l'Italia, a Banti (2000). 
fra le posizioni avanzate da Alessandro Verri, sul Caffé $e^{3}$, agli inizi degli anni '60, da Cesarotti negli anni '80, e da Denina durante il suo soggiorno berlinese (vedi Marazzini 1989: 127-165), e quelle cui giungono invece, agli inizi degli anni '90, lo stesso Alessandro Verri, Galeoni Napione, e in forma molto più appassionata e drammatica Alfieri, sin dalla prima stesura della Vita scritta da esso. A differenza del fratello Pietro, che rimarrà fedele fino all'ultimo alle proprie giovanili posizioni cosmopolite, Alessandro si orienta negli anni romani verso una difesa sempre più rigida e patriottica della tradizione letteraria italiana, e opta per un netto rifiuto della lingua francese $\mathrm{e}^{4}$. Nel settembre 1795, quando ormai si diffonde il timore di un'imminente invasione francese, egli si lamenta che «l'Italiano [...] non forma una nazione, né una potenza», che «la nostra maggior gloria sono i Musici Castrati» (Verri 2008: 973). Come ultimo baluardo contro lo sfacelo totale, rimane solo la lingua «letteraria» italiana (Verri 1806: xiii e xxii-xxiii) ${ }^{5}$.

In modo simile al Caffé, ma con più grande coerenza e sistematicità di pensiero, Cesarotti elaborava a sua volta una concezione dinamica della lingua - e una visione moderna e cosmopolita delle relazioni linguistiche e culturali fra le nazioni - nel Saggio sulla filosofia delle lingue applicato alla lingua italiana (1800), pubblicato una prima volta nel 1785 con titolo di Saggio sulla lingua italiana. Come spiega limpidamente Marazzini (1998: 318-324), per Cesarotti tutte le lingue nascono e si trasformano, e sono il prodotto composito di varie influenze, non di un progetto razionale unitario: nessuna quindi è mai pura. Tutte possono inoltre perfezionarsi, nessuna è così abbondante da non potersi ulteriormente arricchire. Cesarotti (1969: 83) giunge su queste basi, ma in modo teoricamente più rigoroso, a conclusioni non molto diverse da quelle del giovane Verri: «Se la lingua francese ha dei termini appropriati ad alcune idee necessarie che in Italia mancan di nome, $[. .$.$] per quale strano e ridicolo aborrimento ricuserem di$ accettarle? [...] Qual insensato patriottismo ci fa dunque sdegnare i frutti stranieri che possono esserci d'alimento e delizia? ${ }^{6}$.

Ma proprio queste sono le tesi contro cui insorge Galeani Napione, pubblicando nel 1791 Dell'Uso e dei pregj della lingua italiana. Anche di questo autore ciò che interessa in questa sede è soprattutto il sistema

${ }^{3}$ Alludo qui alla famosa Rinunzia avanti notaio [...] al Vocabolario della Crusca (1764).

${ }^{4}$ Per uno studio minuzioso di questa evoluzione rimando al volume recente di $\mathrm{Mu}-$ sitelli (2016: 286-296).

${ }^{5}$ Citato da Musitelli (2016).

${ }^{6}$ Sulla concezione cesarottiana del 'genio di una lingua' e del 'carattere di una nazione', molto più plastica e dinamica di quella di un Condillac, vedi anche Gambarota (2011: 177-189). 
metaforico, che fa della credenza in un carattere nazionale, della fedeltà alla lingua italiana e del rifiuto del bilinguismo un imperativo etico, e quasi un precetto di morale sessuale. Lo scrittore osserva sin dalle prime pagine della sua opera che proprio «noi di Piemonte», che «siam posti ai confini d'Italia [...] dovremmo esser zelantissimi di conservar l'original nostro carattere incorrotto, escludendo l'uso delle lingue straniere, che il modo di pensare, e le opinioni straniere porta seco infallantemente» (Galeani Napione 1791: xvi-xvii). «I climi, i costumi, le lingue - egli aggiunge - sono mura di divisione, che assai meglio di quella famosa de' Cinesi, separano, e distinguono le nazioni» (Galeani Napione 1791: 4). Riprendendo alla fine del primo libro lo stesso tema, Napione osserva che «la natura medesima col frapporre tra noi e le oltramontane nazioni gli asprissimi gioghi delle alpi [...] coll'ispirarci in cuore gli Italiani sentimenti, [...] col renderci oltremodo difficile l'uso della lingua Francese, Italiani ci vuole, ed alla lingua Italiana ci chiama». Per cui solo «la tirannia della moda» e «l'avversione a' costumi nazionali» ci può spingere «a spogliare $[. .$.$] l'indole nostra per vestirne una$ straniera» (Galeani Napione 1791: 98).

Lo scrittore constata che per non pochi piemontesi l'italiano è la «lingua di gravità», la «consorte», il francese «la lingua di vezzo», «la favorita», che si pratica «per diletto». Ma non c'è allora rischio - egli chiede - che «si raffreddino gli affetti [...] verso la legittima sposa?» (Galeani Napione 1791: 54). Proseguendo sulla stessa falsariga, egli paragona il latino a un «cavallo sfrenato che trasporta lo Scrittore dove gli pare», e il francese a un «ginnetto gentile», al cui governo basta il «placido impero di una mano anche femminile». L'italiano lo paragona invece a «un corsiero forte, brioso, instancabile, come quelli de' Palladini de' Romanzi», animali «feroci e alteri», che «non obbediscono, se non se alla mano degli eroi» (Galeani Napione 1791: 172-173). Se ne può dedurre che la lingua italiana è la sola che esiga locutori autenticamente virili: e allora capiamo perché anche Alessandro Verri la prescrivesse come terapia a una nazione italiana che aveva ormai come unica gloria i cantori castrati.

Ma veniamo ad Alfieri, il cui caso è forse il più interessante e rivelatore. Alla fine degli anni '60, l'astigiano è ancora vicino a posizioni cosmopolite come quelle di Cesarotti. Egli viaggia con passione inesausta per tutta Europa, sogna una carriera nella diplomazia, si entusiasma per il teatro francese (Alfieri 1997: 62-63, 64, 66, 67, 69). Ma tra il 1773 e il 1775 si compie in lui una radicale trasformazione, quella che chiama la sua «conversione letteraria e politica» (Alfieri 1997: 63), una riforma a un tempo linguistica, intellettuale e morale, ma che in certi momenti è addirittura descritta in termini fisiologici, come una trasformazione quasi somatica. A proposito delle lezioni d'inglese cui si era sottoposto durante il suo primo soggiorno fiorentino, Alfieri ricorda per esempio con vergogna come «barbaramente 
andasse balbettando» questa lingua a lui così ostica. Ma si consola pensando che almeno, già allora, si era «ripurgata la pronunzia» dall' «orribile U lombardo, o francese», che ti costringe - per pronunciarlo - a una "smorfia" risibile come quella "che fanno le scimmie allorché favellano» (ibidem). L'intera filosofia illuminista risulta in tal modo derisa, dal momento che è impossibile pronunciarne il significante supremo («nature») senza alterare la boccuccia come una scimmia, o come fanno i raffinati gentiluomini francesi - precisa Alfieri - quando soffiano sulla minestra.

La conversione alle lettere risulta altrettanto tormentata sul piano delle conquiste lessicali e concettuali. Alfieri è infatti costretto a riconoscere che nel momento in cui iniziava la sua rivoluzione intellettuale e morale, egli non solo scriveva, ma propriamente pensava in francese; e confessa allora la propria «angoscia di dover fare versi italiani di pensieri francesi» (Alfieri 1997: 82). Il risultato, almeno in un primo tempo, sono dei testi anfibi, che vengono a trovarsi «fra il francese e l'italiano, senza essere né l'una cosa né l'altra». Per liberarsi di una lingua «spiacevole e meschina» come il francese egli deve allora invasarsi di modi toscani, deve spensare per poi ripensare, dando il «bando a ogni qualunque lettura francese» (Alfieri 1997: 81). Ma come appropriarsi delle ricchezze della lingua italiana senza fare indigestione di versi antichi, masticati e rimasticati senza più neppure intenderne il senso? Alfieri tiene duro solo perché spera che alla fine tutte queste forme e parole esotiche di cui si è inondato il cervello memorizzando versi di Dante, Petrarca e Tasso si immedesimeranno coi suoi pensieri ed affetti, e si trasformeranno in qualcosa che sia veramente suo.

La conversione di Alfieri alle lettere segna dunque la nascita di un paradigma nazionale, di una concezione patriottica della letteratura, resa più aggressiva negli ultimi anni della sua vita dal misogallismo, e rilanciata all'inizio del XIX secolo da scrittori come Cuoco e Foscolo. Nel celebre Saggio storico sulla rivoluzione di Napoli $(1801,1806)$ ritroviamo le stesse metafore, lo stesso rigido sistema binario che già abbiamo incontrato in Verri, Alfieri e Galeani Napione: "Quel nobile sentimento di orgoglio che solo ispira le grandi nazioni [...] era interamente estinto presso di noi. Noi divenimmo a vicenda or Francesi, or Tedeschi, ora Inglesi; noi non eravamo più nulla. [...] La manìa per le nazioni estere prima avvilisce, indi immiserisce, finalmente ruina una nazione spegnendo in lei ogni amore per le cose sue» (Cuoco 2014: 22-23).

Quanto a Foscolo, egli suggerisce nell'Ortis che le passioni del suo eroe - l'amore della madre, delle lettere e della patria, l'amore di una casta fanciulla la cui felicità è sacrificata dal padre ai propri interessi patrimoniali - non siano che le diverse figure di un'unica vocazione. Secondo il paradigma che abbiamo visto affiorare in diversi autori, coloro che regolano il proprio comportamento sul modello della società parigina e dei romanzi 
francesi, non solo sono incostanti nei loro amori, sono anche incapaci di servire la patria e di perseverare nella virtù. Jacopo, invece, è fedele fino alla morte alla donna che non gli è dato sposare, e ama con la stessa devozione la lingua, la storia e la letteratura italiana: piuttosto che andare a mendicare un «pane straniero» in Francia, preferisce darsi la morte nei colli Euganei, presso la donna che ama, e farsi inumare nella terra che vide nascere $\mathrm{i}$ suoi antenati. Il testamento spirituale che affida ai posteri è costituito da un pacchetto di lettere che esaltano amore e virtù, denunciano il dominio straniero e esortano le generazioni future a realizzare ciò che nel presente è impossibile: ridare all'Italia libertà, unità e indipendenza.

Con Foscolo giunge insomma a piena articolazione un sistema amoroso, linguistico, letterario e politico coerente, che si diffonderà rapidamente nella prima metà del XIX secolo e svolgerà un ruolo fondamentale sia in Italia che in tutta l'Europa delle nazioni, e al di là.

\section{CARATTERE NAZIONALE E SCAMBI FRA LE CULTURE IN MADAME DE STAËL}

Non c'è dubbio che il paradigma appena descritto abbia avuto un forte impatto anche su Madame de Staël. La scrittrice si dissocia, sin dalle prime pagine di De la littérature, dal classicismo di Voltaire, Marmontel o La Harpe, che avevano ribadito la fissità e l'universalità del bello e del gusto, e sottolinea contro di loro le differenze caratteristiche che ci obbligano a distinguere le opere degli autori secondo le loro diverse nazionalità - italiane, inglesi, tedesche o francesi (de Staël 2017a: 13) ${ }^{7}$. Secondo de Staël, queste differenze costituiscono una norma, un valore, attraverso il quale una nazione acquista un'identità che non ha ancun rapporto con la forza militare e le azioni guerriere: la guerra - osserva la scrittrice - permette di conquistare un territorio rapidamente. Ma per trasformarlo in nazione ci vuole ben altro: bisogna inculcare negli abitanti che vi soggiornano gli stessi gusti, gli stessi sentimenti, le stesse abitudini, e le armi per raggiungere questo scopo non sono fucili e cannoni, ma eloquenza e filosofia, amore delle lettere e delle arti (de Staël 2017a: 27).

Ne risulta, secondo de Staël, che una nazione non può avere un carattere se non è libera. Lo prova fra l'altro il caso italiano. Pur lodando negli italiani il magico incanto della loro brillante immaginazione, de Staël osserva che in Italia la divisione politica, la moltitudine dei partiti, delle fazioni e dei principati, hanno finito col depravare i caratteri, che l'amor di patria avrebbe reso invece più grandi, se la nazione si fosse unita sotto un

${ }^{7}$ Sul concetto di nazionalità in Madame de Staël e in tutto il gruppo di Coppet vedi già Marcelli (1977). 
solo governo (de Staël 2017a: 116). Essa constata in Italia un eccesso di voluttà, dovuto all'influenza delle belle arti, del sole e di una lingua molle e armoniosa, per cui ormai gli italiani preferiscono occupare il primo rango nei piaceri piuttosto che il secondo nella gloria (de Staël 2017a: 124-125). De Staël si oppone per questo all'imitazione della letteratura italiana nei paesi settentrionali. Le bellezze che rendono immortali i poeti italiani dipendono infatti dalla lingua, dal clima, dall'immaginazione meridionale, da circostanze che non si possono trasportare altrove. I loro difetti sono invece contagiosi, e quindi, se le nazioni settentrionali non fossero protette dalle loro passioni profonde e dall'atmosfera brumosa delle terre in cui vivono - che esige dall'individuo una straordinaria forza morale - l'affettazione italiana li corromperebbe rapidamente (de Staël 2017a: 122).

Anche de Staël, come Herder, ritiene che si debba cercare in un popolo il suo tratto caratteristico, ciò che ne costituisce l'essenza più autentica. Nei popoli settentrionali questo tratto è l'attaccamento all'indipendenza, conseguenza del fatto che il clima ostile, e la malinconia nativa che ne deriva, li priva di ogni altra consolazione o compensazione: le nazioni del Nord amano tanto la libertà perché questa è la loro prima e unica felicità (de Staël 2017a: 130).

Dieci anni dopo, in De l'Allemagne, ritroviamo nelle grandi linee le stesse idee. De Staël ricorda di nuovo l'importanza delle credenze che cementano una nazione: ella osserva per esempio che una nazione può facilmente accontentarsi dei beni comuni della vita, la quiete e una certa agiatezza. Ma per costituire una patria ci vogliono beni più nobili: «Le sentiment patriotique se compose des souvenirs que les grands hommes ont laissés, de l'admiration qu'inspirent les chefs-d'œuvre du génie national; enfin de l'amour que l'on ressent pour les institutions, la religion et la gloire de son pays» (de Staël 1968, I: 80).

Da queste stesse premesse deriva l'atteggiamento della scrittrice nella famosa querelle des anciens et des modernes. De Staël ha una concezione dinamica della letteratura, essa ha fede nella "perfectibilité humaine», e questo è uno dei motivi per cui non crede che i moderni possano andare a scuola dagli antichi, e si oppone alle varie forme di classicismo e di regolismo. Ma in più essa pensa che per noi moderni le letterature antiche siano delle letterature trapiantate, come le letterature straniere, e siano quindi incapaci di inseminare la nostra cultura. Per de Staël, l'unica letteratura che sia veramente connaturata a noi moderni, che abbia delle radici nel nostro suolo, che meriti di essere coltivata, è la letteratura cavalleresca: «elle exprime notre religion; elle rappelle notre histoire: son origine est ancienne, mais non antique» (de Staël 1968, I: 213-214).

Da qui anche il principale difetto che essa rimprovera alla letteratura francese. Siccome è la più classica fra le moderne, è anche la sola che non 
si sia diffusa nel popolo: le stanze del Tasso sono cantate dai gondolieri veneziani, Shakespeare è ammirato sia dal popolo che dai nobili, i poeti francesi sono invece apprezzati solo dalle persone colte di tutta Europa (de Staël 1968, I: 214). E manca quindi alla Francia un poema nazionale come quello dei Nibelunghi, a cui hanno contribuito il patriottismo, la religione, la vita di un popolo nel corso dei secoli, e in cui sono rappresentati eventi immensi, che il poeta trova depositati nella memoria collettiva della nazione: «Les personnages du poème épique doivent représenter le caractère primitif de la nation» (de Staël 1968, I: 218-219). Nell'epos dei Nibelunghi si ritrova lo stampo indistruttibile da cui è uscita tutta la storia tedesca, in esso si riflette ciò che è bello e grande nel suo carattere:

Ce qu'il y avait de beau en Allemagne, c'était l'ancienne chevalerie, sa force, sa loyauté, sa bonhomie et la rudesse du Nord qui s'alliait avec une sensibilité sublime. Ce qu'il y avait aussi de beau, c'était le christianisme enté sur la mythologie scandinave, cet honneur sauvage que la foi rendait pur et sacré; [...] cet enthousiasme de la mort, ce paradis guerrier où la religion la plus humaine a pris place (de Staël 1968, I: 229).

È triste pensare - come ci ricorda oggi l'opera di Anselm Kiefer, che spesso rimanda a De l'Allemagne - che Madame de Staël abbia potuto contribuire alla diffusione di quella mitologia cristiana e guerriera del sangue e del sacrificio, che alimenterà per un secolo e mezzo il nazionalismo e il militarismo tedesco e culminerà nei deliri nazisti. Se ce n'era bisogno, abbiamo qui un'altra prova di quanto sia aleatoria la nozione di carattere nazionale, e di quanto sia arbitrario trarne delle conclusioni sul destino dei popoli. De Staël ha sottovalutato i rischi insiti nel nazionalismo e dell'eroismo guerriero, non ha riconosciuto la sua inconciliabilità con la morale umanista, e non è stata capace di anticipare la trasformazione del patriottismo in egoismo nazionale e in imperialismo. E tuttavia è chiaro che niente le era più estraneo dell'espansionismo militare e del culto della violenza, niente le ripugnava quanto il sangue sparso gratuitamente, quello che era stato per esempio versato in Francia durante la Rivoluzione, e dagli eserciti di Napoleone in Europa. Per lei l'eroismo guerriero non è mai un fine a se stesso, ma un mezzo per risollevare una nazione depressa, e che essa dovrà poi temperare con la cultura, le lettere e altre istituzioni pacifiche, una volta riconquistata la libertà.

A questo riguardo, dobbiamo constatare un' anomalia, una dissonanza rivelatrice. De Staël considera la Francia come sua patria (cfr. de Staël 1996: 135), anche se come noto era figlia di genitori ginevrini, di austera religiosità protestante. E tuttavia essa difende solo occasionalmente la tradizione letteraria francese, e sostiene invece con grande vigore il diritto delle altre letterature a difendersi dall'influenza straniera. De Staël riprende molti degli 
argomenti attorno ai quali si stava costruendo il nazionalismo tedesco, ma sarebbe un errore considerarla come una nemica del proprio paese, come la riteneva Napoleone. Era anzi un'ammiratrice sincera della propria letteratura, anche se Schiller era riuscito a farla dubitare della superiorità del sistema drammatico francese (vedi per esempio de Staël 1968, I: 195). E ciò che ammirava nella cultura tedesca non era il progetto egemonico che stava nascendo in quegli anni in Germania, ma proprio la sua resistenza all'egemonia francese (cfr. Winock 2012: 487-488) ${ }^{8}$. Così infatti leggiamo nell'ultimo capitolo di De l'Allemagne: «je n'aurais pu parler de la guerre avec enthousiasme sans me la représenter comme celle d'une nation libre combattant pour son indépendance» (de Staël 1968, II: 311n). Gli ultimi tre capitoli di quest'opera sono un elogio dell'entusiasmo 9 , che è nello stesso tempo una polemica contro lo scetticismo e l'egoismo dei Lumi, una risposta alle polemiche dell'illuminismo tedesco contro la Schwärmerei. Per de Staël, l'entusiasmo è il sentimento più nobile, ma proprio perché è il sentimento provato da chi difende la propria patria: «que le signal se fasse entendre, que la bannière nationale flotte dans les airs, et vous verrez des regards jadis si doux, [...] tout à coup animés par une volonté sainte et terribile!» (de Staël 1968, II: 311). Questo è anche il senso dell'apostrofe che chiude l'opera, in cui l'amore per la Francia («terre de gloire et d'amour!») brilla di viva luce, e l'entusiasmo è nuovamente contrapposto al calcolo e all'ambizione conquistatrice, che crede di conculcare il mondo ma in realtà si limita a trasformare in deserto le terre più fertili (de Staël 1968, II: 316).

Alla luce di queste ultime osservazioni, non potrà quindi sorprenderci che accanto ai passi numerosi in cui de Staël sembra far propria una concezione autoctona della nazione, ve ne siano altri da cui si ricava l'impressione che il suo vero scopo sia piuttosto combattere il nazionalismo culturale e politico, e incoraggiare la mutua fecondazione delle culture e delle nazioni.

Così, per esempio, nella prefazione alla seconda edizione di De la littérature, dopo avere ammesso che esiste un gusto francese, e che non si possono introdurre in Francia tutte le incoerenze dei tragici inglesi e tedeschi, de Staël aggiunge che bisogna essere del tutto insensibili al genio

${ }^{8}$ Si veda anche l'acuta osservazione di Starobinski (1966: 1057): «L'Allemagne, destinée au public français au moment où l'hégémonie française couvre toutes les autres voix, a pour but principal non d'exalter une nationalité rivale, mais de marquer la nécessité d'une attention à ce qui est radicalement autre. Mme de Staël a fort bien vu que la fidélité à soi-même, dont se prévalent tous les nationalismes, devient inféconde si elle ne se double d'un égard profond pour l'étranger et pour la parole étrangère».

${ }^{9}$ Sulla concezione staëliana dell'entusiasmo, vedi Amend (1994) e la riflessione più generale di Pearson (2016: 158-296) sul ruolo civile attribuito da Staël alla donna scrittrice, che educa i cittadini e fonda lo stato con la pietà e l'entusiasmo. 
per non ammirare quanto vi è di appassionato e profondo negli scrittori del Nord (de Staël 2017a: 6). Di conseguenza, bisogna certo studiare gli autori antichi e conoscere perfettamente i classici del secolo di Luigi XIV, ma se si presentasse questo secolo come un modello di perfezione oltre al quale nessun scrittore eloquente potrà mai sollevarsi, la Francia rinuncerebbe per sempre a possedere in futuro grandi scrittori (de Staël 2017a: 7) ${ }^{10}$. La stessa idea è formulata in De l'Allemagne attraverso il rifiuto di una metafora che già aveva usato, ma in chiave positiva, Galeani Napione, e che sarà ripresa anche da Corinne: «Nous n'en sommes pas, je crois, à vouloir élever autour de la France littéraire la grande muraille de la Chine, pour empêcher les idées du dehors d'y pénétrer» (de Staël 1968, I: 47; cfr. anche de Staël 2017b: 1134).

Venendo poi a parlare delle invasioni barbariche e della caduta dell'impero romano, de Staël osserva come quelle invasioni - che pure produssero tante rovine - permisero agli abitanti di più di metà dell'Europa, rimasti fino ad allora esclusi dalla civiltà, di cominciare ad approfittarne (de Staël 2017a: 45). Le popolazioni snervate del Mezzogiorno, mescolandosi con quelle del Nord, ne trassero nuova energia, e a loro volta trasmisero ai loro conquistatori una duttilità mentale che completava le loro facoltà intellettuali (de Staël 2017a: 95). Secondo de Staël questa fusione di nazioni diverse fu nello stesso tempo una sorta di invasione delle classi superiori da parte di popolazioni più rozze, e nel presente, con la Rivoluzione francese, assistiamo forse a un processo analogo: in un primo tempo, esso è accompagnato da esplosioni di cieca violenza, che suscitano orrore e spavento, ma col tempo potrà contribuire alla nascita di una nuova civiltà (de Staël 2017a: 100).

Un analogo effetto benefico produce secondo de Staël la mescolanza fra uomini e donne, incoraggiata dal cristianesimo. Nell'antichità, quando le donne erano equiparate agli schiavi e le amicizie erano solo maschili, tutte le virtù avevano come fondamento l'amor di patria. Ma nella donna la pietà verso i deboli, la simpatia per chi soffre, l'elevazione dell'anima contano più delle virtù propriamente politiche; ed è cosi che i moderni hanno scoperto la filantropia (de Staël 2017a: 108-109). Fra gli antichi l'obbedienza filiale era la suprema virtù, fra i moderni è più stimato l'amore paterno (de Staël 2017a: 111). Il più grave crimine non è più provare dolore, ma esserne causa. E avere paura della morte non è più una vergogna, dal momento che ogni vita umana è diventata sacra.

Per de Staël, insomma, le nazioni dovrebbero essere energiche e combattive verso gli stati nemici che vogliono conquistarle, ma rispettose della libertà degli stati più deboli; esse dovrebbero essere fiere delle proprie

${ }^{10}$ Così ripeterà anche Corinne, rivolgendosi al tipico rappresentante del classicismo francese, il conte d'Erfeuil (de Staël 2017b: 1124-1125). 
tradizioni nazionali ma anche aperte a influenze che permetteranno loro di rinnovarsi. Gli scambi artistici e culturali fra le nazioni sono un formidabile strumento di civiltà e di progresso. Niente riassume con maggior forza la sua posizione a questo riguardo quanto l'incipit del saggio uscito nel 1816 in italiano, nella versione di Pietro Giordani, nel primo numero della $\mathrm{Bi}$ blioteca italiana:

Trasportare da una ad altra favella le opere eccellenti dell'umano ingegno è il maggior benefizio che far si possa alle lettere; perchè sono sì poche le opere perfette, e la invenzione in qualunque genere è tanto rara, che se ciascuna delle nazioni moderne volesse appagarsi delle ricchezze sue proprie, sarebbe ognor povera: e il commercio de' pensieri è quello che ha più sicuro profitto (de Staël 1943: 3) ${ }^{11}$.

\section{CORINNE OU L'ITALIE}

Accostiamoci ora a Corinne. Non c'è dubbio che uno dei grandi temi del romanzo sia la differenza fra le nazioni e i loro caratteri, il contrasto fra le personalità degli individui appartenenti a nazioni diverse, e dunque l'impossibilità dell'amore e della felicità coniugale fra persone che non hanno la medesima patria. Oswald, il protagonista maschile dell'opera, è presentato sin dalle prime pagine come un giovane scozzese ${ }^{12}$ che ama teneramente il padre e la patria, ma si rimprovera, prima ancora di incontrare Corinne, di averli, almeno una volta, amaramente traditi. Il padre si era infatti ammalato mentre Oswald era lontano da lui, trattenuto a Parigi da una relazione amorosa con una francese, nonostante il suo paese fosse ormai in guerra con la Francia (de Staël 2017b: 1241, 1251). Scoprendo, al suo ritorno in Gran Bretagna, che il padre non era più in vita, si era rimproverato di avere accorciato i suoi giorni, e si era convinto che il genitore fosse morto vedendo in lui un ribelle alla patria e al volere paterno, a tutto ciò che è sacro su questa terra (de Staël 2017b: 1006). Il suo stato di salute e una morbosa malinconia lo spingono ora a cercare distrazione e sollievo in Italia. Ma proprio il fatto di allontanarsi dall'Inghilterra, attraversando il mare, aumenta i suoi sensi di colpa: «Tout est solennel dans un voyage dont l'Océan marque les premiers pas: il semble qu'un abîme s'entrouvre derrière vous, et que le retour pourrait devenir à jamais impossible» (de Staël 2017b: 1007).

${ }^{11}$ Cfr., per l'originale francese, de Staël (2013: 595). Sull'importanza della teoria staëliana della traduzione, vedi Bann (1994).

${ }^{12}$ Simone Balayé osserva che probabilmente de Staël ha fatto di Oswald uno scozzese per prudenza politica, ma che di fatto, in tutto il romanzo, la scrittrice non fa nessuna distinzione fra nobiltà inglese e scozzese (in de Staël 2000: 2n). 
Si aggiunga che Oswald nutre verso l'Italia dei pregiudizi in cui si riflettono alcune delle opinioni della stessa Madame de Staël intorno al carattere degli italiani ${ }^{13}$ : il giovane ha vissuto a Londra dove gli interessi politici assorbono tutti gli altri, e in Francia, dove la società è tutto. Ma per apprezzare le meraviglie della natura e i capolavori dell'arte italiana, non basta quell' «esprit de jugement» cui mira l'educazione nel suo paese d'origine, ci vuole anche molta immaginazione, una facoltà che in Oswald è rimasta allo stato embrionale (de Staël 2017b: 1020). Egli è stato abituato sin dall'infanzia ad amare «l'ordre et la prospérité publique». Avvicinandosi a Roma, è colpito dal fatto che intere regioni siano scarsamente abitate, che la terra sia incolta e negletta, e biasima l'indolenza degli abitanti e dei loro capi (ibidem).

Parallelamente, Corinne si presenta a Oswald come una poetessa che improvvisa i suoi versi in scena, un'artista le cui origini sono ignorate da tutti, e che si muove con sicurezza in quella sfera pubblica da cui le donne 'oneste' sono in genere escluse, in Inghilterra in modo particolare, dove il loro campo d'azione è ristretto alla vita domestica. Oswald è sin dall'inizio ammaliato dalla sua bellezza e dal suo talento, ma prova anche diffidenza nei suoi confronti. E Corinne, da parte sua, si esprime come un'ardente patriota italiana. Durante la sua prima apparizione in pubblico, in occasione della sua incoronazione poetica in Campidoglio, essa canta un inno all'Italia, alle sue glorie passate e alle sue speranze future - un inno non molto diverso da quello che in quegli anni potevano scrivere Foscolo o altri poeti italiani. L'invocazione che apre il carme attribuisce all'Italia un primato sia culturale sia militare: «Italie, empire du soleil; Italie, maîtresse du monde, berceau des lettres» (de Staël 2017b: 1030). La poetessa prosegue la sua improvvisazione affermando che Roma conquistò l'universo col suo genio, e fu regina con la libertà, che poi l'invasione dei barbari, distruggendo il paese, precipitò nelle tenebre l'intero universo. Più tardi, tuttavia, l'immaginazione ha restituito all'Italia, attraverso le arti e le lettere, quel primato che essa non poteva più esercitare nel campo politico (de Staël 2017b: 1030-1034).

Nonostante la reciproca, forte attrazione che provano Corinne e Oswald, affiorano quindi sin dall'inizio gli ostacoli che si oppongono al loro amore. Il principe Castel-Forte, amico generoso e fedele, la mette subito in guardia, mostrando lucida preveggenza:

Ses goûts n'ont pas le moindre rapport avec les vôtres. Vous ne vous en apercevrez pas tant qu'il sera sous le charme de votre présence, mais votre empire sur lui ne tiendrait pas, s'il était loin de vous. [...] et vous savez d'ailleurs combien les An-

${ }^{13}$ Sull'evoluzione del pensiero di Staël riguardo all'Italia rimane essenziale Balayé (1971), di cui si vedranno in particolare i capitoli dedicati al viaggio in Italia (93-259). Vedi anche Alfonzetti \& Bellucci (2010). 
glais en général sont asservis aux mœurs et aux habitudes de leur pays (de Staël 2017b: 1055) $)^{14}$.

Poco dopo scopriamo infatti che Oswald sospetta Corinne di frivolezza nelle sue relazioni amorose, si chiede se sia capace di un amore esclusivo come quello che una donna deve provare, secondo lui, per il proprio marito, si domanda se sia stata già innamorata di altri. Dimenticando che lui stesso ha avuto una lunga relazione con una donna che lo ingannava, egli vorrebbe sposare un' adolescente innocente e casta, che conocendolo si schiudesse per la prima volta all'amore (de Staël 2017b: 1058). E più tardi infatti, incontrando Lucile, che ha appena compiuto 16 anni, si commuoverà pensando alla purezza celestiale di una fanciulla che non si è mai allontanata dalla madre, che ha conosciuto della vita solo la tenerezza filiale (de Staël 2017b: 1348). Alle insinuazioni di Oswald, Corinne risponde orgogliosamente che una donna sensibile non può giungere ai 26 anni senza avere conosciuto le illusioni dell'amore; ma che non è certo sua colpa se non ha ancora incontrato un uomo che meritasse il suo affetto (de Staël 2017b: 1058).

L'incomprensione fra i due aumenta quando Oswald comincia a sospettare che Corinne conosca bene l'Inghilterra: se vi avete vissuto a lungo - le chiede - come avete potuto lasciare quel santuario del pudore e della delicatezza, per venire a vivere in questo paese, dove non soltanto la virtù, anche l'amore è ignoto? Quale poeta italiano - prosegue Oswald - ha scritto versi paragonabili a quelli di Thompson che, nel suo canto della primavera, dipinge con tratti così commoventi la felicità dell'amore nel matrimonio? «Y a-t-il un tel mariage en Italie? Et là où il n'y a pas de bonheur domestique, peut-il exister de l'amour?» (de Staël 2017b: 1106).

Sarebbe tuttavia un errore credere che solo Oswald resista a Corinne e diffidi di lei, mentre lei sarebbe disposta a tutto per amor suo. In realtà l'idea di un matrimonio che la obbligherebbe a vivere in Inghilterra e a rinunciare alle sue attività artistiche e letterarie spaventa Corinne, la quale ha sofferto molto durante gli anni trascorsi nel perduto Northumberland, affidata alle 'cure' di lady Edgerton, la sua severa matrigna ${ }^{15}$.

Da qui tutta una serie di atti mancati, o di reazioni di orgoglio eccessivo, che la spingono a rimandare le nozze, anche quando l'occasione se ne presenta. Quando, per esempio, i due giovani salgono su un vascello inglese che si trova nel porto di Napoli, Corinne si immagina per un momento che Oswald voglia portarla davanti a un sacerdote e prenderla come sposa, e la

${ }^{14}$ L'idea sarà reiterata più tardi dalla stessa Corinne (de Staël 2017b: 1306).

${ }^{15}$ La donna le ripetava in continuazione: «Une femme est faite pour soigner le ménage de son mari et la santé de ses enfants», discrezione e pudore sono le sue principali virtù, e se ha del talento deve celarlo (de Staël 2017b: 1279). 
sua prima reazione - di cui più tardi si pentirà amaramente - non è di gioia ma di spavento (de Staël 2017b: 1222). In un momento successivo, Corinne chiede a Oswald se prenderla in moglie non sarà per lui un sacrificio, se egli, dopo averla sposata, non rimpiangerà l'anima pura di Lucile, a cui l'aveva destinata suo padre. E quindi gli confessa che se la cosa fosse possibile, preferirebbe passare la vita accanto a lui, ma senza un legame coniugale (de Staël 2017b: 1297).

Alla luce di queste premesse, non può sorprenderci che l'amore di Corinne e Oswald sia destinato a naufragare. Certo, a un primo livello del testo, i due non si sposano a causa di una serie di contrattempi che la scrittrice sembra avere escogitato, senza una vera necessità interna, al solo scopo di accrescere la suspense e prolungare il romanzo. Ma a un livello più profondo, de Staël sembra pensare che anche se le circostanze fossero state più fortunate, un matrimonio fra Oswald e Corinne non sarebbe stato comunque possibile.

Oswald aveva promesso a Corinne che, se fosse venuto a sapere che il padre non si era opposto esplicitamente al loro matrimonio, l'avrebbe sposata, ma lei sarebbe dovuta venire a vivere con lui in Inghilterra, adottando i costumi dell'isola, e i compiti che vi sono assegnati alla donna (de Staël 2017b: 1303). Se invece avesse scoperto che il genitore si era espresso contro le loro nozze, non l'avrebbe sposata, ma sarebbe venuto a vivere accanto a lei in Italia, accontentandosi di un ruolo di confidente e di amico (de Staël 2017b: 1339). Al suo ritorno in Inghilterra, Oswald viene informato dell'impressione negativa che Corinne aveva fatto su suo padre, quando l'aveva incontrata (de Staël 2017b: 1360-1362), e quindi rinuncia a sposarla, ma non mantiene la sua promessa: non torna in Italia, sposa invece Lucile e provoca in tal modo la crisi che condurrà Corinne alla tomba quattro anni dopo. Tuttavia, se anche non avesse violato la parola data, sembra poco probabile che i due sarebbero stati una coppia felice. Il ritorno di Oswald avrebbe probabilmente ridato vita e energia a Corinne, avrebbe risvegliato il suo genio poetico. Ma Oswald sarebbe stato scontento della propria esistenza, perché invece di mettere il proprio ingegno al servizio di qualche nobile causa, avrebbe dovuto accontentarsi dell'esistenza indolente e inutile del sigisbeo, del pariniano «giovin signore» ${ }^{16}$.

Ragioni profonde li privano dunque dalla felicità coniugale, e sono le ragioni che ho esplorato sin dall'inizio di questo saggio. Siamo agli inizi del XIX secolo: in tutta Europa si sta diffondendo il senso dell'appartenen-

${ }^{16}$ Proprio questo è il motivo per cui il padre di Oswald è contrario al suo matrimonio con Corinne: «Quel sort pour un habitant de nos montagnes, que de traîner une vie oisive au sein des plaisirs de l'Italie! un Écossais sigisbé de sa femme, s'il ne l'est pas de celle d'un autre!» (de Staël 2017b: 1361; il corsivo è nel testo). Cfr. Gengembre (1999: 104-105). 
za nazionale; le lingue, le letterature e le nazioni europee si concepiscono sempre più come entità separate, incommensurabili e spesso nemiche. L'ampio ritorno a una concezione patriarcale della famiglia impone inoltre alle donne, anche nobili, di accontentarsi dei ruoli domestici. Non è quindi verosimile, benché i loro due paesi non siano propriamente in guerra, che lo scozzese Nelvil e l'italiana Corinne - che solo fuggendo dall'Inghilterra ha potuto sottrarsi a una vera e propria 'morte civile' - possano unirsi e amarsi per tutta una vita. Mettendo in scena questa impossibilità, de Staël prende atto della realtà storica, riconosce l'influenza di un canone patriottico e di genere che anche lei condivide in gran parte ${ }^{17}$.

Rimane tuttavia una domanda. Se Oswald e Corinne sono così diversi, perché si amano, perché si sentono attratti l'uno dall'altro con tanta forza, e dunque che senso ha, se ha un senso, la loro passione? A questa domanda, si potrà rispondere in primo luogo che ciò che li affascina mutualmente è proprio il contrasto dei loro caratteri. Come si è visto, Oswald ha un'innata tendenza alla malinconia, tratto di carattere tipico delle nazioni settentrionali - abituate da una natura ostile a raccogliersi in se stesse, a contemplare gli abissi del proprio cuore - e che nel suo caso è aggravato dai rimorsi che prova nei confronti del padre. Proprio per questo motivo egli diffida del clima italiano, del carattere allegro e vivace degli italiani, di quelle che gli sembrano le prove della loro superficialità e frivolezza. Ma nello stesso tempo la sensibilità italiana lo attira in quanto antidoto contro la malinconia, e sin dall'inizio è colpito dalla straordinaria vitalità di Corinne.

Risvegliandosi a Roma il giorno in cui la poetessa deve essere incoronata in Campidoglio, vedendo il sole che brilla in un cielo sereno, e ascoltando il suono lieto delle campane che suonano a distesa in tante chiese della città, egli si sente penetrato da un sentimento di amore e riconoscenza (de Staël 2017b: 1022). Poco dopo, quando Corinne conclude la sua prima improvvisazione affermando che il clima italiano consola anche dalle pene del cuore, Oswald è l'unico a non applaudire: l'affermazione della poetessa contraddice infatti una delle convinzioni più profonde della sua anima malinconica (de Staël 2017b: 1034). Tuttavia Corinne, vedendolo così triste, indovina i suoi pensieri, sente la giustezza del suo rimprovero e quindi, riprendendo la parola, cerca una risposta alla sua inquietudine. Essa ammette che neppure il cielo italiano può sciogliere certe pene; ma osserva che proprio a Roma, a causa delle sue tante tombe e rovine, la malinconia

${ }^{17}$ Cfr. Garry-Boussel (2000: 65): «Corinne est un roman consacré aux barrières sociales, culturelles, linguistiques et sexuelles qui divisent irréductibilement les hommes: l'auteur élève un mur imaginaire entre les peuples du Nord et les peuples du Midi [...] pour mieux abattre ce rempart de préjugés et d'incompréhension». Sul tema delle diversità nazionali in Corinne vedi anche Rosset (2000). 
può destare nell'anima un'impressione più dolce che in altri luoghi. I popoli del mezzogiorno sono penetrati dal pensiero della morte non meno degli altri popoli. Ma essi riescono a riconciliare la morte con l'immaginazione, riescono a rappresentarsi la fine dell'esistenza con colori meno tenebrosi degli abitanti del Nord. In una città come Roma «le soleil comme la gloire réchauffe même la tombe», e così un'armonia più dolce si mescola all'esistenza (de Staël 2017b: 1035).

Con queste parole, che commuovono Oswald, Corinne risponde a un suo intimo desiderio. È vero infatti che lei è nata in Italia e si sente italiana; ma parla perfettamente l'inglese, suo padre era inglese, ha vissuto a lungo in Inghilterra e proprio in Inghilterra è maturato il suo talento poetico. Avendo vissuto in entrambi i paesi, essa di fatto racchiude in sé le doti di tutte e due le nazioni, e questo rende la sua personalità più profonda e più variegata, benché anche più tormentata. Così essa scrive a Oswald, a proposito del suo lungo soggiorno in Inghilterra:

Mon talent que j'avais craint de perdre s'était accru par l'étude suivie que j'avais faite de la littérature anglaise; la manière profonde de penser et de sentir qui caractérise vos poètes avait fortifié mon esprit et mon âme, sans que j'eusse rien perdu de l'imagination vive qui semble n'appartenir qu'aux habitants de nos contrées. Je pouvais donc me croire destinée à des avantages particuliers par la réunion des circonstances rares qui m'avaient donné une double éducation, et, si je puis m'exprimer ainsi, deux nationalités différentes (de Staël 2017b: 1289).

Non a caso quindi, sin dal loro primo incontro, Oswald prova meraviglia e ammirazione davanti a Corinne: «Oswald était tout à la fois surpris et charmé, inquiet et entraîné, il ne comprenait pas comment une seule personne pouvait réunir tout ce que possédait Corinne» (de Staël 2017b: 1043). In un'altra occasione, egli critica la sua scelta di essere italiana, ma poi riconosce che essa ha una grazia incomparabile proprio perché riunisce in sé «les charmes qui caractérisent les différentes nations» (de Staël 2017b: 1105-1106). Non potrà quindi meravigliarci che le parole di Corinne suscitino in Oswald una speranza di guarigione, che egli sia attirato dai benefici che lui, uomo del Nord, potrebbe trarre dall'unione con una personalità solare, meridionale, ma non insensibile alla malinconia nordica: «Que serait-ce donc s'il pouvait à la fois retrouver les souvenirs de sa patrie, et recevoir par l'imagination une vie nouvelle, renaître pour l'avenir, sans rompre avec le passé!» (de Staël 2017b: 1038).

Un evento successivo ci mostra di nuovo quanto questa sua doppia natura arricchisca la personalità di Corinne. Per dimostrare che il teatro italiano potrebbe in avvenire risorgere, essa traduce in italiano Romeo e Giulietta e recita in pubblico la tragedia, ottenendo uno straordinario successo, che rivela l'originalità del genio di Shakesperare, ma anche quello della sua 
traduttrice. Shakespeare è infatti un autore inglese (de Staël 2017b: 1106). Ma secondo de Staël questa pièce è stata scritta con quell'immaginazione del Mezzogiorno, a un tempo appassionata e ridente, che trionfa nella felicità, ma passa, non meno rapidamente, dalla felicità alla disperazione e dalla disperazione alla morte (de Staël 2017b: 1138). Rigida adepta del carattere individuale delle nazioni, de Staël ammette qui che un poeta possa scrivere con la sensibilità di un altro paese, che possa essere nello stesso tempo inglese e italiano. E infatti agli spettatori entusiasti sembra che la tragedia di Shakespeare, tradotta da Corinne in italiano, sia rientrata nel'alveo della sua lingua materna (de Staël 2017b: 1139) ${ }^{18}$.

Ma naturalmente, anche un'altra ragione ci spiega perché Corinne abbia scelto di tradurre e di recitare proprio Romeo e Giulietta. In questa tragedia si riflette infatti il dramma personale di Corinne e di Oswald. Romeo e Giulietta non possono coronare il loro sogno d'amore perché appartengono a due famiglie rivali, che si oppongono alla loro unione. In modo simile, anche per Corinne e per Oswald le differenze fra le due nazioni di cui fanno parte sono un ostacolo insormontabile al loro amore: «Too early seen unknown and known too late» (de Staël 2017b: 1139) ${ }^{19}$, dice Giulietta (e ripete in scena Corinne), quando scopre che Romeo è membro della famiglia rivale. Analogamente, Oswald scopre di non avere diritto di amare Corinne quando essa gli rivela le sue origini, che fino ad allora gli aveva celato, e viene a sapere dell'opposizione di suo padre alle loro nozze (de Staël 2017b: 1360). Ma ormai è troppo tardi, Corinne morirà della sua passione, e Oswald rimarrà minato per sempre dal rimorso e dalla malincolia.

Di converso, l'amore di Corinne per Oswald simboleggia il rinnovamento che avverrebbe in Italia, se le leggi e le istituzioni inglesi potessero dare un po' di stabilità e disciplina al genio italiano. La voce narrante evita di presentarci Corinne come un'allegoria dell'Italia. E tuttavia leggiamo l'elogio che fa di lei in Campidoglio il principe di Castel-Forte, perspicace osservatore delle società umane. Grazie allo studio approfondito delle letterature straniere - egli dice - Corinne unisce nel modo più compiuto l'immaginazione, i quadri, la vita brillante del Mezzogiono, insieme con quell'osservazione del cuore umano che sembra la prerogativa esclusiva dei paesi che sono meno interessati agli oggetti esteriori (de Staël 2017b: 1026):

${ }^{18} \mathrm{Su}$ questa problematica, si vedano i due saggi di Pouzoulet (1999a: 80-83 e soprattutto 1999b). Considerazioni analoghe, con riferimento alla canzone della Mignon goethiana, parafrasata da Corinne in una delle sue improvvisazioni in Campidoglio, già figuravano in Bosse (1988).

${ }^{19}$ Romeo and Juliet, a. I, sc. 5, v. 136 (ed. S. Greenblatt). 
Regardez-la - egli aggiunge - c'est l'image de notre belle Italie; elle est ce que nous serions sans l'ignorance, l'envie, la discorde et l'indolence auxquelles notre sort nous a condamnés; nous nous plaisons à la contempler comme une admirable production de notre climat, de nos beaux-arts, comme un rejeton du passé, comme une prophétie de l'avenir (de Staël 2017b: 1028-1029).

Capiamo allora perché nell'epilogo del romanzo il giudizio della voce narrante su Oswald, che ha sposato una fanciulla del suo paese, e non l'affascinante e misteriosa straniera di cui si era invaghito viaggiando in Italia, sia così diverso da quello che troviamo in tante opere scritte, come Corinne, nella prima metà del XIX secolo. In Die Jungfrau von Orleans (1802) di Schiller, la vergine guerriera vede annientata all'improvviso la sua potenza il giorno in cui, durante la battaglia, al momento di colpire il giovane inglese Lionel, «elle se sent tout à coup attendrie par sa figure, et l'amour entre dans son cœur» (così l'autrice riassume l'azione in de Staël 1968, I: 306). Essersi innamorata del proprio nemico, questa è la colpa che Johanna si rimprovera amaramente, e che più tardi potrà espiare solo morendo in battaglia. De Staël, in De l'Allemagne, riproduce lunghi passi del suo lamento: «Malheureuse! Dieu veut un instrument aveugle; c'est avec des yeux aveugles que tu devais obéir. Tu l'as regardé, c'en est fait, la paix de Dieu s'est retirée de toi, et les pièges de l'enfer t'ont saisie» (de Staël 1968, I: 307-308). Nel Guillaume Tell di Rossini (1829) - che a sua volta s'ispira a una pièce di Schiller - lo svizzero Arnoldo si rimprovera l'amore che prova per la principessa austriaca Matilde, da lui salvata da una valanga, e la folle speranza che lo ha indotto a servire per amore di lei nell'esercito austriaco. Ha così tradito i compagni e la patria, che gli ordinano l'odio e la guerra contro i nemici del suo paese ${ }^{20}$. Ma quando Guglielmo e Gualtiero gli rivelano che il governatore austriaco, il tirannico Gessler, ha fatto ammazzare suo padre, egli rinuncia per sempre a Matilde (a. II, sc. 4).

L'amore di Anna per il sovrano ottomano, nel Maometto II di Rossini (1820), quello di Norma per il governatore romano nell'opera di Bellini (1831), sono altri esempi di un riprovevole amore per il nemico, spesso messo in scena nei drammi e nei melodrammi romantici. In molte di queste opere, amare il nemico, e esporsi per questo al rischio di tradire la patria, sembra essere il peccato supremo, che solo la morte potrà espiare. Come si è visto, anche in Corinne si trova qualche traccia di un patriottismo così esclusivo, dal momento che Oswald è in un certo senso punito per essersi lasciato sedurre da una francese dall'anima ignobile, Madame d'Arbigny, proprio mentre la sua patria era in guerra contro la Francia. Ma la nota dominante, negli ultimi due capitoli del romanzo, sono i sensi di colpa che

${ }^{20}$ Gioacchino Rossini, Guillaume Tell, mélodrame tragique en 4 actes, livret d'Etienne de Jouy et Hippolyte Bis, a. I, sc. 4 e 5. 
prova Oswald verso Corinne, per non essere stato capace di costanza nel proprio amore, per essere stato fuorviato dai suoi pregiudizi contro l'Italia e le donne italiane, e dal testamento del padre. A differenza di Arnold, Johanna e Norma, la cui colpa è di avere amato lo straniero, il nemico, la colpa di Oswald è di non averlo saputo amare abbastanza. Il suo torto è di non avere capito fino in fondo quale straordinaria ricchezza sia l'amore fra persone di nazioni diverse, quale meravigliosa occasione sia l'incontro fra le culture.

\section{BIBLIOGRAFIA}

Alfieri, V. (1997). Vita di Vittorio Alfieri da Asti scritta da esso, a cura di S. De Stefanis Ciccone e P. Larson. Viareggio-Lucca: Mauro Baroni.

Alfonzetti, B. \& Bellucci, N. (a cura di). (2010). Corinne e l'Italia di Mme de Staël. Roma: Bulzoni.

Amend, A. (1994). Le système de l'enthousiasme d'après Mme de Staël. In K. Kloocke e S. Balayé (a cura di), Le groupe de Coppet et l'Europe (1789-1830) (pp. 269-290). Lausanne et Paris: Institut Benjamin Constant et Jean Touzot.

Balayé, S. (1971). Les carnets de voyage de Madame de Staël. Contribution à la genèse de ses ceuvres. Genève: Droz.

Bann, S. (1994). Théorie et pratique de la traduction au sein du Groupe de Coppet. In K. Kloocke e S. Balayé (a cura di), Le groupe de Coppet et l'Europe (1789-1830) (pp. 217-233). Lausanne et Paris: Institut Benjamin Constant et Jean Touzot.

Banti, A. M. (2000). La nazione del Risorgimento: parentela, santità e onore alle origini dell'Italia unita. Torino: Einaudi.

Banti, A. M. (2005). L'onore della nazione. Torino: Einaudi.

Bosse, M. (1988). Corinne ou l'Italie, diagnostic d'un dilemme historique.

In M. Matucci (a cura di), Il gruppo di Coppet e l'Italia (pp. 83-107). Pisa: Pacini.

Cesarotti M. (1969). Saggio sulla filosofia delle lingue, a cura di M. Puppo. Milano: Marzorati.

Crépon, M. (1996). Les géographies de l'esprit: enquête sur la caractérisation des peuples de Leibniz à Hegel. Paris: Payot.

Cuoco, V. (2014). Saggio storico sulla rivoluzione di Napoli, a cura di A. De Francesco. Roma-Bari: Laterza.

De Staël, G. (1943). Sulla maniera e l'utilità delle traduzioni. In E. Bellorini (a cura di), Discussioni e polemiche sul Romanticismo (1816-1826) (Vol. I, pp. 3-9). Bari: Laterza. 
De Staël, G. (1968). De l'Allemagne (2 vol), a cura di S. Balayé. Paris: Garnier-Flammarion.

De Staël, G. (1996). Dix années d'exil, a cura di S. Balayé e M. Vianello Bonifacio. Paris: Fayard.

De Staël, G. (2000). Corinne ou l'Italie, a cura di S. Balayé. Paris: Champion.

De Staël, G. (2013). De la littérature et autres essais littéraires, a cura di S. Genand. Paris: Champion.

De Staël, G. (2017a). De la littérature considérée dans ses rapports avec les institutions sociales. In G. De Staël, Euvres (pp. 1-303), a cura di C. Seth e V. Cossy. Paris: Gallimard "La Pléiade".

De Staël, G. (2017b). Corinne ou l'Italie. In G. De Staël, Euvres (pp. 10031460), a cura di C. Seth e V. Cossy. Paris: Gallimard "La Pléiade". Galeani Napione, G. (1791). Dell'uso e dei pregj della lingua italiana libri tre con un discorso intorno alla storia del Piemonte. Vol. I. Torino: Gaetano Balbino.

Gambarota, P. (2011). Irresistible Signs. The Genius of Language and Italian National Identity. Toronto: University of Toronto Press.

Garry-Boussel, C. (2000). L'homme du Nord et l'homme du Midi dans Corinne. In M. Delon e F. Mélonio (a cura di), Mme de Staël (pp. 55-66). Paris: Presses de l'Université de Paris-Sorbonne.

Gengembre, G. (1999). Corinne, roman politique. In S. Balayé (a cura di), L'éclat et le silence. Corinne ou l'Italie de Madame de Staël (pp. 81-108). Paris: Champion.

Herder, J. G. (1994). Schriften zu Philosophie, Literatur, Kunst und Altertum 1774-1787. Frankfurt am Main: Deutscher Klassiker Verlag.

Hume, D. (1987). Of National Characters. In D. Hume, Essays, Moral, Political, and Literary (pp. 197-215), a cura di E. F. Miller. Indianapolis: Liberty Fund.

Marazzini, C. (1989). Storia e coscienza della lingua in Italia dall'Umanesimo al Romanticismo. Torino: Rosenberg \& Sellier.

Marazzini, C. (1998). La lingua italiana. Profilo storico [1994]. Bologna: il Mulino.

Marcelli, U. (1977). Il gruppo di Coppet e il concetto di nazionalità. In S. Balayé e J.-D. Candaux (a cura di), Le groupe de Coppet (pp. 401-415). Genève/Paris: Slatkine/Champion.

Musitelli, P. (2016). Le flambeau et les ombres. Alessandro Verri, des Lumières à la Restauration. Roma: École française de Rome.

Pearson R. (2016). Unacknowledged legislators: The poet as Lawgiver in Post-Revolutionary France: Chateaubriand-Staël-Lamartine-Hugo - Vigny. Oxford: Oxford University Press.

Pouzoulet, C. (1999a). Pour une renaissance politique et littéraire de l'Italie: enjeux du modèle de Dante chez Madame de Staël et Sismondi. In 
J.-P. Perchellet (a cura di), Madame de Staël Corinne ou l'Italie (pp. 71-86). Paris: Klincksieck.

Pouzoulet, C. (1999b). Corinne ou l'Italie: à quoi sert un roman pour penser l'Italie en 1807. In S. Balayé (a cura di). L'éclat et le silence. Corinne ou l'Italie de Madame de Staël (pp. 39-79). Paris: Champion.

Rivarol, A. de (1995). Discours sur l'universalité de la langue française. In Académie de Berlin, De l'universalité européenne de la langue française. 1784. Paris: Fayard.

Rosset, F. (2000). Poétique des nations dans Corinne ou l'Italie. In C. Planté et al. (a cura di), "Une mélodie intellectuelle». Corinne ou l'Italie de Germaine de Staël (pp. 139-158). Montpellier: Publications de l'université Paul-Valéry.

Starobinski, J. (1966). Mme de Staël et la définition de la littérature. La nouvelle Revue Française, 168, 1054-1059.

Verri, A. (1806). Alessandro Verri editore agli amatori dell'italiana letteratura. In I quattro libri di Senofonte dei Detti memorabili di Socrate (pp. V-XXIII). Brescia: Nicolò Bettoni.

Verri, P. e Verri, A. (2008). Carteggio di Pietro e Alessandro Verri. Vol. II, a cura di S. Rosini. Roma: Edizioni di Storia e Letteratura.

Winock, M. (2012). Madame de Staël [2010]. Paris: Fayard/Pluriel.

\author{
MADAME DE STAËL'S CORINNE OU L'ITALIE: THE IMPASSE \\ OF CULTURAL AND ROMANTIC MEETING BETWEEN 'NATIONS' \\ IN THE EARLY $19^{\text {TH }}$ CENTURY EUROPE
}

\title{
Sum mary
}

Madame de Staël's position on the question of national character is to be placed within the context of a debate involving many European intellectuals since the middle of the 18th century - a debate in which language, literature and politics are strictly linked. In her major works, from De la littérature to De l'Allemagne, Madame de Staël puts forward ideas that despite their global coherence hint at subtle distinctions and tensions. In Corinne, namely, the contrast between national characters becomes an obstacle to dialogue that even the most authentic love between the individuals cannot be overcome by. However, even in this novel dialogue is reasserted by Madame de Staël as a positive, although unaccomplished ideal, against retreat into one's national identity.

Keywords: Madame de Staël, National character, Patriotism, Encounter between cultures, Representation of love, Corinne ou l'Italie 